\title{
American Society of Hematology 2020 Podcast Collection: MDS and AML
}

António Almeida

Received: February 25, 2021 / Accepted: February 26, 2021 / Published online: March 22, 2021

(c) The Author(s) 2021

\section{PODCAST TRANSCRIPT}

Victoria Glasson (VG): Managing Editor of Advances in Therapy

António Almeida (AA): Head of Department, Hospital da Luz, Lisbon, Portugal

VG: Hello, and welcome to the Adis Rapid+ series. We're bringing you a selection of podcasts covering the American Society of Hematology (ASH) 2020 Conference, discussing the highlights of the data released.

Today's podcast will be focusing on MDS (myelodysplastic syndrome) and AML (acute myeloid leukemia), and speaking to us today is Professor António Almeida, Head of Department at Hospital da Luz in Lisbon, Portugal, and also head of the Hemato-oncology Diagnostic Lab in the Portuguese Institute of Oncology in Lisbon. Thank you so much for joining us today, António.

AA: Not at all. Hi, Victoria, good afternoon.

VG: So you're going to take us through your top highlights of the MDS and AML data that was released at the ASH conference, the implications of this data on future developments. A

Supplementary Information The online version contains supplementary material available at https:// doi.org/10.1007/s12325-021-01691-0.

A. Almeida $(\square)$

Hospital da Luz, Lisbon, Portugal

e-mail: amalmeida@hospitaldaluz.pt lot of important data was released at the conference, so what are your main take-home messages from the conference? And can you tell us what, in your opinion, are the most exciting breakthrough data at the conference?

AA: Well, I think this is a really exciting time for both MDS and AML. And if I could have one take-home message, it is that the future, the very near future, will bring us a lot of exciting new treatments. Really what has happened in these fields is that we have gone from having what I would call the machine gun approach in which we tried to kill all the leukemic chemicals and hope that the normal bone marrow regenerates, to having a much more targeted approach in identifying new molecular lesions, identifying new targets that are far more efficacious, and which treatment is far less toxic than traditional approaches.

So perhaps if I could start along these molecular lines of how much they've contributed first of all, to diagnosis. And looking at MDS, the diagnosis is not always very obvious. I mean, we're normally investigating patients with cytopenias, but there are multiple causes for cytopenias and we do not always have an immediate answer.

And the diagnosis of MDS relies heavily on morphology, which sadly, in many countries, is becoming a dying art. So we need sort of stronger indicators. And the Cleveland group did a very elegant study in collaboration with 
two Italian groups, looking at different markers that could predict MDS [1]. These markers include simple factors such as white cell count, neutrophil count, eosinophil count. And also more sophisticated markers such as which genes are mutated, which somatic mutations are identified. And based on this, they were able to design an algorithm that highly increases the likelihood and the probability of accurately diagnosing MDS.

So I think this is really where the future is going. I do not think morphology is going to go out altogether, but I think we're going to have more precise diagnostic tools that will help us distinguish MDS from other causes of cytopenias, and give a more precise diagnosis to our patients.

Following along from this, in the low-risk MDS group, we had some very exciting data looking at new treatment options. The first study that was presented, which I find very interesting, is a study of lenalidomide in patients with deletion 5q MDS who are not yet transfusion dependent [2].

Now classically, we wait for these patients to be transfusion dependent before we start lenalidomide treatment. But the Sintra-Rev study looked at patients who had deletion $5 \mathrm{q}$ MDS, who were not transfusion dependent, but had anemia. So they had some symptoms-they weren't altogether asymptomatic, but they did not need transfusions-and started them on low-dose lenalidomide, $5 \mathrm{mg}$, and compared another similar cohort treated with placebo. What they showed is that the patients [who] were starting on the lenalidomide had a much longer time to transfusion dependence and a much longer time with normal hemoglobins than those who were treated with placebo. So this does indicate that there is an advantage in pre-emptive treatment of these patients.

Another exciting development was the oral azacitidine presentation. This trial had many problems, especially with the population that they chose. The population was a very, if I may call it, high-risk end of low-risk MDS [3]. There were patients who had transfusion dependent, low-risk patients who have thrombocytopenia. Indeed, this was a particularly poor risk group, so much so that there was an increased mortality during the screening period, so even before we started treatment. But even so, this trial showed that these patients had an increased transfusion independence rate which lasted for about 1 year when they were treated with the oral azacitidine. So this could be a promising new step in the future.

And again, the imetelstat data, which is a telomerase inhibitor, also showed very promising results with a high rate of transfusion independence in non-deletion $5 \mathrm{q}$ patients who were transfusion dependent, and some of these patients really went into complete remission [4]. So in the low-risk group, I think we have exciting new treatments. And all sorts of exciting new ways of treating our patients, treating them earlier and having pre-emptive responses.

The other side-and this sort of crosses over also into the AML population-are the high-risk patients. Now traditionally, these patients were either candidates for transplant or not. And those who were not candidates for transplant generally were treated with a hypomethylating agent. And for a long time, very little progress was made in improving the prognosis of these patients.

I think what was really exciting at this year's conference is that at least three new developments have come along which hold real promise for these patients. The first development is venetoclax. Venetoclax has been tested both in MDS and AML, so it's transversal [applicable] to both groups [5]. And in combination with azacitidine, it has produced very impressive response rates of up to $90 \%$ overall response rates, which are durable, and which actually overtake [increase] the survival of patients to about 1 year. So this is a very exciting development.

This is obviously an inhibition of apoptosis and with a high transfusion independence rate. So again, I think this will be very much there in the future. And I think many people like us, in Portugal, are starting to use this routinely for high-risk MDS and for AML.

But the exciting new treatment options were monoclonal antibodies. Magrolimab, which is an anti-CD47 antibody which basically inhibits that self-signal that leukemic cells have [6]. So by using magrolimab, leukemic cells are not 
recognised to self, and therefore are attacked by the immune system.

And this antibody was used in combination with azacitidine, with a hypomethylating agent, and this had very impressive response rates; overall response rates of over $90 \% ; 42 \%$ complete remission rates; and an overall survival in the small group-this is a phase 1B, 33 patients-but even so, an overall survival which was not reached in median. So this is very promising after median follow-up with more than a year.

Another very promising agent is pevonedistat. This inhibits NEDD8. Again, NEDD8 is an anti-apoptopic signal. So basically, we are again increasing apoptosis and autophagy by using pevonedistat. And again, we've got very impressive response rates, over $80 \%$ overall response rate, and very impressive overall survivals, with the combination of pevonedistat and azacitidine having almost 2 years' overall survival compared to 1 year with azacitidine alone [7]. And so these are two different agents which really have improved the results on azacitidine.

And finally, I'd like to highlight the results with MBG-453. Again, used in both high-risk MDS and AML. So these two areas really complement each other. And so sabatolimab, which is an anti-TIM-3 antibody, produced overall response rate of $64 \%$. But the attractive part is that it inhibits TIM-3, and by inhibiting TIM-3, it certainly stimulates apoptosis, but it also stimulates activation of the immune system against these cells [8].

So with all these new targets and all these new agents, I think we're really moving forward to a much more targeted therapy era. And I think this is what is really exciting in MDS and in AML. We're identifying molecular targets. We're identifying subgroups that respond better to different treatments. And we're having nonchemotherapeutic options, which really favour the population that we're treating, which is mainly elderly patients.

So I really hope that in the future, we'll be able to have some sort of algorithm by which we identify specific characteristics of patients, and they will have a much more personalized treatment, much more efficacious and less toxic than we can offer nowadays.

VG: You've mentioned about modelling, and possibly algorithms, and it does point to the potential for us to have sort of artificial intelligence and the use of these algorithms really overtaking morphology, which is currently the gold standard for diagnosis. But taking over, and possibly having AI and computer modelling as the gold standard for diagnostics, but also predicting treatment options for patients. Do you think that's an option?

AA: Absolutely. And along those lines, there was an interesting paper presented at the congress in which, using the same computer algorithms, the Cleveland group looked at the probability of response with different characteristics. In this sense, they used several characteristics of the disease to see whether patients responded to azacitidine or not [9]. And I'm sure the same strategy can be used to see whether patients will respond to these new agents.

I think artificial intelligence is very important. We collect an enormous amount of data in these trials. Not only molecular data, but clinical data, side-effect data, and it's very difficult to manually be able to identify co-existing factors that predict responses. So I think artificial intelligence will go a long way to developing algorithms that predict response, that identify best treatments for which patients based on the series, the multitude of data, that we can collect easily from patients.

VG: That's really interesting. And something else as well, that I noticed from the conference, was the US study group that showed that there could arguably be no age cut-off for transplants in older patients with MDS because they've been shown to benefit from stem cell transplantation [10]. And this can open up an additional treatment option for a group of patients that are not frequently offered stem cell transplants. Are you able to talk about that at all?

AA: Yeah, absolutely. And I think, again, that is a very exciting development. You know, I think, par and par with what we've had in terms of developing novel treatment options with more efficacy and with fewer serious side effects, is the fact that we can improve the aggressive treatment options as we've had with transplant. 
The current transplant is a reduced intensity, of being able to use hyperidentical donors with reasonable toxicity. And being able to use transplant techniques with less toxicity of the older population, combined with the fact that we are able to induce responses in a much larger proportion of population that we transplant, I think will open up the door to a lot more treatments. Because despite my inevitable excitement at the new treatments, it is undeniable that the survivals are still around 2 years. And that, if you are a patient with 70, 75 years of age, is not very long at all.

So if we are able to use these novel treatments to induce deeper remissions and then to use transplant to be able to effectively cure the disease, I think we'll be doing a huge service to this population.

VG: Excellent. And then just speaking to that as well, you mentioned before about the more personalizing, the treatments that are really looking at the specific mutations and treating them, rather than the chemotherapies which are the more traditional treatment options, but they have very limited specificity for the different mutations when you stratify patients. That's going to definitely impact upon patient quality of life, as well as overall survival. Would you agree?

AA: Yes. I think the novel therapies have huge advantage of being less toxic than intensive chemotherapy. Now, I think we also must be realistic and realize that intensive chemotherapy will still have a place, and there is a population, proportion of population, that will benefit.

So despite our understandable excitement at new therapies, and new targets, and new ways of doing things, I also think that we need to have a sober mind and realize that a lot of our older treatments are still there, they're still available, and the patients will still benefit. The important thing is to identify which patients benefit the most from which treatments.

VG: Thank you so much, António, for speaking with us today, and for taking us through your round-up of the AML and MDS highlights in the conference. I hope this will have been really helpful for all of our listeners, giving a great summary of the ASH conference.
Please look out for the other podcasts in this ASH 2020 coverage collection.

You can listen to more podcasts by subscribing to Adis Rapid+ podcast with your preferred podcast provider. A full list of declarations, including funding and all the disclosure statements, can also be found on the journal website.

\section{DIGITAL FEATURES}

This article is published with digital features, including a podcast video and audio file, to facilitate understanding of the article. To view digital features for this article go to https://doi. org/10.6084/m9.figshare.14113457.

\section{ACKNOWLEDGEMENTS}

Funding. This podcast has been developed independently through an unrestricted educational grant by Novartis. The Rapid Service Fee and Open Access publication was funded by an unrestricted educational grant by Novartis.

Authorship. All named authors meet the International Committee of Medical Journal Editors (ICMJE) criteria for authorship for this article, take responsibility for the integrity of the work as a whole, and have given their approval for this version to be published.

Disclosures. António Almeida has received speaker fees from Novartis, BMS, Alexion and Abbvie.

Compliance with Ethics Guidelines. This article does not contain any studies with human participants or animals performed by any of the authors.

Peer Review. Please note, contrary to the journal's standard single-blind peer review process, as a podcast this article underwent review by a member of the journal's Editorial Board. 
Open Access. This article is licensed under a Creative Commons Attribution-NonCommercial 4.0 International License, which permits any non-commercial use, sharing, adaptation, distribution and reproduction in any medium or format, as long as you give appropriate credit to the original author(s) and the source, provide a link to the Creative Commons licence, and indicate if changes were made. The images or other third party material in this article are included in the article's Creative Commons licence, unless indicated otherwise in a credit line to the material. If material is not included in the article's Creative Commons licence and your intended use is not permitted by statutory regulation or exceeds the permitted use, you will need to obtain permission directly from the copyright holder. To view a copy of this licence, visit http://creativecommons.org/licenses/bync/4.0/http://creativecommons.org/licenses/ by-nc/4.0/.

\section{REFERENCES}

1. Goll JB, Jensen TL, Lindsley RC, et al. O540: Targeted sequencing of 7 genes can help reduce pathologic misclassification of MDS. ASH 2020 conference. 2020. https://ash.confex.com/ash/ 2020/webprogram/Paper140317.html.

2. Cadenas FL, Lumbreras E, Xicoy B, et al. O536: Phase 3 study of lenalidomide (LEN) vs placebo in non-transfusion dependent (TD) low risk $\operatorname{del}(5 \mathrm{q})$ MDS patients - interim analysis of the European Sintra-Rev Trial. ASH 2020 conference. 2020. https://ash.confex.com/ash/2020/webprogram/ Paper140339.html.

3. Garcia-Manero G, Santini V, Almeida A, et al. A phase III placebo-controlled trial of CC-486 in patients with red blood cell transfusion-dependent (RBC-TD) anemia and thrombocytopenia due to IPSS lower-risk myelodysplastic syndromES (LRMDS). EHA Library. Garcia-Manero G. 06/12/20; 295000; S180. https://library.ehaweb.org/eha/2020/ eha25th/295000/guillermo.garcia-manero.a.phase. iii.placebo-controlled.trial.of.cc-486.in.html?f= listing\%3D0\%2Abrowseby\%3D8\%2Asortby\%3D2\% 2Asearch\%3Dblast.
4. Platzbecker U, Fenaux P, Steensma DP, et al. O658: Treatment with imetelstat provides durable transfusion independence (TI) in heavily transfused non$\operatorname{del}(5 q)$ lower risk MDS (LR-MDS) relapsed/refractory $(\mathrm{R} / \mathrm{R})$ to erythropoiesis stimulating agents (ESAs). ASH 2020 conference. 2020. https://ash. confex.com/ash/2020/webprogram/Paper140985. html.

5. Garcia JS, Wei AH, Borate U, et al. O656: Safety, efficacy, and patient-reported outcomes of venetoclax in combination with azacitidine for the treatment of patients with higher-risk myelodysplastic syndrome: a phase $1 \mathrm{~b}$ study. ASH 2020 conference. 2020 . https://ash.confex.com/ash/2020/ webprogram/Paper139492.html.

6. Sallman DA, Asch AS, Kambhampati S, et al. O330: The first-in-class anti-CD47 antibody magrolimab combined with azacitidine is well-tolerated and effective in AML patients: phase $1 \mathrm{~b}$ results. ASH 2020 conference. 2020. https://ash.confex.com/ ash/2020/webprogram/Paper134728.html.

7. Sekeres MA, Watts JM, Radinoff A, et al. O653: Efficacy and safety of pevonedistat plus azacitidine vs azacitidine alone in higher-risk myelodysplastic syndromes (MDS) from study P-2001 (NCT02610777). ASH 2020 conference. 2020. https://ash.confex.com/ash/2020/webprogram/ Paper135840.html.

8. Brunner AM, Esteve J, Porkka K, et al. O657: Efficacy and safety of sabatolimab (MBG453) in combination with hypomethylating agents (HMAs) in patients with acute myeloid leukemia (AML) and high-risk myelodysplastic syndrome (HR-MDS): updated results from a phase $1 \mathrm{~b}$ study. ASH 2020 conference. 2020. https://ash.confex.com/ash/ 2020/webprogram/Paper136855.html.

9. Radakovich N, Meggendorfer M, Malcovati L, et al. O541: A personalized clinical-decision tool to improve the diagnostic accuracy of myelodysplastic syndromes. ASH 2020 conference. 2020. https:// ash.confex.com/ash/2020/webprogram/ Paper139412.html.

10. Nakamura R, Saber W, Martens MJ, et al. 0732: A multi-center biologic assignment trial comparing reduced intensity allogeneic hematopoietic cell transplantation to hypomethylating therapy or best supportive care in patients aged 50-75 with advanced myelodysplastic syndrome: blood and marrow transplant clinical trials network study 1102. ASH 2020 conference. 2020. https://ash. confex.com/ash/2020/webprogram/Paper136828. html. 